Discussion Nurse led fibroscan service shows effective monetary benefit in good enhanced care of liver disease. It gives a more accurate diagnosis as compared to USS and also helps in avoiding the invasive liver biopsy in majority of the cases.Utilised in a correct manner it can help in appropriate care of the liver disease patients.

\section{PTU-017 SEPSIS OUTCOMES IN PATIENTS WITH LIVER DISEASE}

Maja Kopczynska*, Tamas Szakmany, Welsh Digital Data Collection Platform Collaborators. Cardiff University School of Medicine, Cardiff, UK

\subsection{6/gutjnl-2019-BSGAbstracts.226}

Introduction Pre-existing liver disease (LD) has long been recognised as a risk factor for the progression of infection to sepsis and increased morbidity and mortality after sepsis episode. Recently it has been questioned what proportion of patient death is directly attributable to sepsis and focus on the role of pre-admission characteristics is emerging. The aim of this study was to investigate the predictive capabilities of sepsis screening tools in sepsis recognition in patients with LD. We also wanted to determine the characteristics, outcomes and proportion of death attributable to sepsis in LD patients.

Methods Secondary analysis of outcomes for patients with LD was performed on patient population recruited into two annual 24-hour prospective point-prevalence studies on the general wards and emergency departments across 15 Welsh hospitals in 2016 and 2017. Inclusion criteria were: patient age $>18$, clinical suspicion of infection and NEWS 33. Patient basic demographics; observations, laboratory results and SIRS, SOFA, qSOFA scores were collected. Patients were followedup for 90 days. Deaths attributable to sepsis were evaluated based on microbiological, radiological and laboratory evidence. Results Out of 839 recruited patients, 24 (2.9\%) had past medical history of LD. 12/24 (50\%) had SIRS score $\geq 2,21 /$ $24(87.5 \%)$ had SOFA score $\geq 2$ and $3 / 24$ (12.5\%) had qSOFA score $\geq 2$. LD patients had higher total SOFA scores than non-LD patients (SOFA score median $4(0-14)$ and $2(0-$ 11 ), respectively, $p=0.001$ ) and $S O F A \geq 2$ was more frequents in $L D$ patients $(p=0.009)$. LD patients were not significantly different than non-LD patients in terms of their frailty score, DNACPR order in place and ceiling of care. There were no significant differences in management of LD in comparison to non-LD patients in terms of Sepsis Six delivery, review by senior clinicians and antibiotic use. Patients with liver disease had lower survival rate $(p=0.028)$ but none of the deaths could be directly attributed to sepsis.

Conclusions It appears that SOFA based criteria is the best screening tool to discover patients with liver disease at risk of sepsis. The 90-day mortality was greater in liver patients than the rest of the population, but we could not find any link between sepsis and outcome, further strengthening previous observations that the mortality attributed to sepsis could be falsely inflated. It appears patient outcomes could be primarily determined by their underlying condition and infection (or the treatment of a potential infection) could have marginal effect on patient outcomes.

\section{PTU-018 ANALYSIS OF BEDSIDE PREDICTORS OF SURVIVAL FOLLOWING TIPSS FOR REFRACTORY ASCITES IN A REGIONAL CENTRE}

Neil Lachlan*, Ruth Gillespie, Stephen Barclay, Ewan Forrest, Adrian Stanley. Nhs Greater Glasgow And Clyde, Glasgow, UK

\subsection{6/gutjnl-2019-BSGAbstracts.227}

Introduction Prognosis in refractory ascites (RA) is poor and patient selection for TIPSS challenging. In 2011 Bureau et al identified Bilirubin $<50 \mathrm{umol} / \mathrm{L}$ and platelet count $>75 \mathrm{x}$ $10^{9} / \mathrm{L}$, the 'Bureau Criteria' (BC), to be predictive of survival post-TIPSS in $73 \%$ vs $31 \%$ in those out with the BC. Age over 65 yrs has also been considered a risk factor for poor outcome post-TIPSS. In this study we analyse the BC and age in a Glasgow cohort.

Methods Retrospective analysis of all patients undergoing TIPSS for RA between 2011-17 in Glasgow was undertaken. Baseline pre-TIPSS data was recorded and actuarial survival at 1 year assessed in 2 groups: those that did, and did not, fulfil the BC. Age $>65$, in isolation of other variables, with 1 year survival was also assessed.

Results 31 patients underwent TIPSS for RA in this 7 year period. Liver disease aetiology was alcohol ( \pm Hepatitis C) in $87 \%$. At baseline pre-TIPSS the mean age was 58.1 yrs (range 36-79), MELD 12.5 (7-21), Bilirubin 30 (5-127) and Platelet count 161 (15-364). 22 patients fulfilled the BC, 2 were transplanted within 1 year of TIPSS. None of the 9 non-BC patients were transplanted. Actuarial 1 year survival was 14/22 $(63.6 \%)$ in the BC group and $3 / 9(33.3 \%)$ in the non-BC group. Of the 6 patients over 65 yrs, all - except 1 who was transplanted - died within 1 year of TIPSS, this was despite 5 of those 6 fulfilling the BC. Mean transplant-free survival in patients over 65 yrs was 4.1 months (Range $0.75-8$ months). Of those that were both $<65$ yrs and fulfilled BC, 1 year actuarial survival reached $70.5 \%$.

Conclusion The combination of bilirubin $<50$ and platelet count $>75$ is predictive of survival post-TIPSS for refractory ascites in our regional cohort and these outcomes are similar to those in the published literature. However, regardless of the bilirubin and platelet count, great caution should be exercised when considering TIPSS in any patient over the age of 65.

\section{PTU-019 REDUCE STUDY: QUALITATIVE OUTCOMES FROM A MULTI-CENTRE MIXED-METHODS FEASIBILITY RCT IN CIRRHOSIS-RELATED PALLIATIVE REFRACTORY ASCITES}

${ }^{1}$ Max Cooper, ${ }^{3}$ Aparajita Pandey, ${ }^{1}$ Alex Pollard, 1,2 Lucia Macken* ${ }^{*}{ }^{4}$ Catherine Evans, ${ }^{2}$ Shani Steer, ${ }^{1,2}$ Ahmed Hashim, 1,2 2 Sumita Verma, ${ }^{2}$ Louise Mason. ${ }^{1}$ Brighton And Sussex Medical School, Brighton, UK; ${ }^{2}$ Brighton And Sussex University Hospitals NHS Trust, Brighton, UK; ${ }^{3}$ Sussex partnership, , UK; ${ }^{4}$ Kings College London, London, UK

\subsection{6/gutjnl-2019-BSGAbstracts.228}

Introduction The REDUCe study is a mixed methods feasibility randomised controlled trial (RCT) comparing palliative long term abdominal drains (LTAD) with standard care, recurrent large volume paracentesis (LVP) in advanced cirrhosis and 